kommen in Einklang. Ueberall, so weit der Mensch bis jetzt vorzudringen vermochte, befindet er sich in einer Atmosphäre, welche ihm den nölhigen Sauerstoff in hinreichender Menge liefert, und der Organismus selbst besitzt durch die Möglichkeit einer Vergrölserung der Berührungsfläche zwischen Gas und Blut und der rascheren Lüftung bis zu einem gewissen Grade noch die Fähigkeit, den Mangel an der zum Leben nolhwendigen Sauerstoffmenge in der äufseren Luft zu ersetzen.

Die beschriebene Versuchsmethode würde noch zur Erörterung mancher interessanter Fragen sich anwenden lassen. So viel sich bis jetzt absehen läst, sind erhebliche Fehler in derselben nicht begründet; bei den vielfachen Modificationen, deren sie fähig ist, und bei der leichten Handhabung, welche sie gestatlet, diurfte ihre ausgedehntere Anwendung zu manchen sowohl für die Physiologie, als namentlich auch für die experimentelle Pathologie wichtigen Thatsachen führen.

\title{
Ueber neue Derivate des Salicylols und des
}

\section{Eugenols;}

\author{
von A. Cahours *).
}

Derivate des Salicylols (Salicylwasserstoffs).

Acetosalicyl. - Bei Einwirkung von Acelylchlorür auf Benzoylwasserstoff in verschlossenen Gefäfsen bildet sich

*) Nach vorläufigen Mittheilungen wurden die auf die Derivate des Salicylols bezüglichen Resultate schon Bd. CIV, S. 109, die auf dio Derivate des Eugenols bezüglichen Bd. CV, S. 263 dieser Annalen 
nach Bertagnini's Versuchen Zimmtsäure, entsprechend der Gleichung :

$$
\mathrm{C}_{14} \mathrm{H}_{6} \mathrm{O}_{2}+\mathrm{C}_{4} \mathrm{H}_{3} \mathrm{O}_{2} \mathrm{Cl}=\mathrm{ClH}+\mathrm{C}_{18} \mathrm{H}_{8} \mathrm{O}_{4} \text {, }
$$

was ganz der Zersetzung entspricht, welche die Zimmtsäure bei Einwirkung von Alkalihydraten erleidet :

$\mathrm{C}_{18} \mathrm{H}_{8} \mathrm{O}_{4}+2(\mathrm{KO}, \mathrm{HO})=\mathrm{C}_{14} \mathrm{H}_{5} \mathrm{O}_{3}, \mathrm{KO}+\mathrm{C}_{4} \mathrm{H}_{3} \mathrm{O}_{3}, \mathrm{KO}+2 \mathrm{H}$.

Die Zersetzung wie die Synthese der Zimmtsäure spricht also dafür, dafs dieselbe eine gepaarle Säure ist, welche die Elemente der Benzoësäure und der Essigsäure enthält und unter Mitwirkung von Wasser diese Säuren wieder bilden kann.

Da zwischen der Cumarsäure und dem Salicylol ähnliche Beziehungen bestehen, wie zwischen der Zimmtsäure und dem Benzoylwasserstoff, so hoffte ich, in entsprechender Weise Cumarsäure in grölserer Menge darstellen und sie einer genaueren Untersuchung unterwerfen zu können. Diese Hoffnung bestäligte sich indessen nicht.

Ich brachte in eine an einem Ende verschlossene, ziemlich weite und etwa 50 Centimeter lange Glasröhre eine Mischung gleicher Volume Salicylol und Acetylchlorür. In der Kälte mischen sich diese beiden Flüssigkeiten ohne auf einander einzuwirken, aber bei dem Erwärmen tritt eine reichliche Entwickelung von Chlorwasserstoff ein und die Mischung wird dickflüssiger. Sowie die Gasentwickelung nachläIst, schinilzt man die Glasröhre zu und erhilzt sie noch einige Stunden lang auf $100^{\circ}$. Lälst man sie dann sehr langsam erkalten, so wird ihr Inhalt zu einer Masse schöner prismatischer Krystalle von bräunlicher Farbe, welche man

kurz angegeben. Wir nehmen aus Cahours' seitdem veröffentlichter ausführlicher Abhandlung : Recherches sur les corps isomères (Ann. ch. phys. [3] LII, 189) die specielleren Angaben über die neuen Verbindungen hier auf.

D. $\mathbf{R}$. 
durch Pressen zwischen Fliefspapier und wiederholtes Umkrystallisiren aus Alkohol reinigt.

Die so erhaltene Substanz ist unlöslich in Wasser, wenig löslich in kaltem, viel reichlicher löslich in siedendem Alkohol ; aus letzterer Lösung scheidet sie sich bei dem Erkalten fast vollständig in schönen Nadeln ab. In Aether ist sie nur wenig löslich. Chlor, Brom und rauchende Salpetersäure wirken energisch auf diese Substanz ein und geben damit krystallisirbare Substitutionsproducte.

Die Analyse dieser Sulstanz ergab die Formel $\mathrm{C}_{18} \mathrm{H}_{8} \mathrm{O}_{6}$ :

\begin{tabular}{|c|c|c|c|c|c|}
\hline & \multicolumn{2}{|c|}{ Gefunden } & & \multicolumn{2}{|c|}{ Berechnet } \\
\hline Kohlenstoff & $6 \overline{6,91}$ & 65,72 & $\mathrm{C}_{18}$ & 108 & 65,85 \\
\hline Wasserstoff & 4,81 & 4,89 & $\mathbf{H}_{8}$ & 8 & 4,78 \\
\hline Sauerstoff & - & - & $\mathrm{O}_{6}$ & 48 & 29,37 \\
\hline
\end{tabular}

Die Zusammensetzung dieser Substanz ist somit die der Cumarsäure, aber die Eigenschaften sind ganz verschieden; beide sind nur isomer. Ich nenne die neue Verbindung Acetosalicyl. Unter denselben Umständen wird die mit dem Salicylol isomere Benzoësäure bei Einwirkung von Acetylchlorür zu einem anderen isomeren Körper, der s. g. wasserfreien Essigsäure-Benzoësäure, welche durch Wasser zu Essigsäure- und Benzoësäurehydrat gespalten wird.

Das Acetosalicyl besitzt eine merkwürdige Beständigkeil. Es kann bei Rothglühhitze über wasserfreien Baryt destillirt werden ohne sich zu zersetzen; ein solches Destillat ergab 66,05 pC. Kohlenstoff und 4,82 Wasserstoff.

Benzosalicyl. - Ich habe in einer vor mehreren Jahren veröffentlichten Abhandlung über die Benzoyl- und die Salicylverbindungen dargethan, dafs das Benzoylchlorïr bei seiner Einwirkung auf Salicylwasserstoff eine der vorhergehenden ähnliche krystallisirbare Substanz bildet, die auch bei der trockenen Destillation von benzoësaurem Kupfer entsleht und 
welche als Parasalicyl benannt wurde. Ich habe diese Substanz nochmals dargestellt und an ihr wieder alle Eigenschaften des Parasalicyls gefunden; eine neue Analyse gab mir folgende Resultate, welche mit denen meiner früheren Versuche übereinstimmen :

\begin{tabular}{lccrrr} 
& gefunden & & \multicolumn{2}{c}{ berechnet } \\
\cline { 3 - 5 } Kohlenstoff & 74,47 & $\mathrm{C}_{28}$ & 168 & 74,33 \\
Wasserstoff & 4,52 & $\mathrm{H}_{10}$ & 10 & 4,44 \\
Sauerstoff & - & $0_{6}$ & 48 & 21,23 \\
\cline { 3 - 5 } & & & & 226 & $100,00$.
\end{tabular}

Toluosalicyl. - Das Toluylchlorür giebt, wie sich erwarten liefs, analoge Resultate. Setzt man zu Salicylol ein gleiches Volum Toluylchlorür, so tritt eine vollständige Mischung beider Flüssigkeiten ohne Wärmeentwickelung oder sonstige besondere Erscheinung ein; aber erhitzt man die Mischung mittelst einiger Kohlen, so erfolgt alsbald lebhafte Einwirkung und Chlorwasserstoff entwickelt sich in reichlicher Menge. Sobald diese Gasentwickelung aufhört, unterbricht man die Operation und lälst dann erkalten. Die Flüssigkeit erstarrt bald zu einer bräunlichen Masse, welche man zwischen Fliefspapier ausprefst und dann mit heifser Kalilauge behandelt, um das unzersetzt gebliebene Salicylol oder das Toluylchlorür zu beseiligen. Wenn das wässerige Kali nichts mehr aufnimmt, wascht man den Rückstand mit siedendem Wasser um iln von Alkali zu befreien, und reinigt ihn zuletzt noch durch wiederholtes Umkrystallisiren aus starkem Alkohol.

Das in dieser Weise dargestellte Toluosalicyl bildet glänzende farblose zerreibliche Prismen, die bei wenig erhöhter Temperatur zu einer farblosen, bei dem Erkalten deutlich krystallinisch erstarrenden Flüssigkeit schmelzen. Es ist unlöslich in kaltem Wasser, wenig löslich in siedendem Wasser und kaltem Alkohol, in ziemlicher Menge löslich 
in siedendem Alkohol, und scheidet sich aus letzterer Lösung in um so gröfseren Krystallen ab, je langsamer dieselbe erkaltet. In Aether löst es sich leichter als in Alkohol. Es zeigt eine sehr grofse Beständigkeit; nicht nur bleibt es bei dem Kochen mit concentrirter Kalilauge unverändert, sondern es läfst sich auch über festes Kalihydrat destilliren ohne Zerselzung zu erleiden. Durch Chlor, Brom und rauchende Salpetersäure wird es unter Bildung krystallisirbarer Producte angegriffen.

Das Toluosalicyl ergab die Zusammensetzung :

\begin{tabular}{|c|c|c|c|c|c|}
\hline \multirow[b]{2}{*}{ Kohlenstoff } & \multicolumn{2}{|c|}{ gefunden } & \multirow[b]{2}{*}{$\mathrm{C}_{30}$} & \multicolumn{2}{|c|}{ berechnet } \\
\hline & 74,95 & $\overline{74,90}$ & & 180 & 75,0 \\
\hline Wasserstoff & 5,10 & 5,07 & $\mathrm{H}_{12}$ & 12 & 5,0 \\
\hline Sauerstoff & - & 一 & $\mathrm{O}_{6}$ & 48 & 20,0 \\
\hline
\end{tabular}

Ich will bei dieser Gelegenheit noch Einiges über das Toluylchlorür mittheilen. Man erhält diese Verbindung sehr leicht nach dem Verfahren, welches ich für die Darstellung solcher organischer Chlorverbindungen im Allgemeinen vor 10 Jahren angegeben habe. Man bringt in eine zweifachtubulirte Retorte äquivalente Gewichtsmengen Toluylsäure und Phosphorsuperchlorid; es ist vortheilhaft, das letztere schwach überschüssig sein zu lassen. Das in der Vorlage sich sammelnde Rohproduct giebt durch wiederholte fractionirte Rectification eine farblose Flüssigkeit von grölserem spec. Gewicht als das des Wassers, die an der Luft raucht und das Licht stark bricht. Durch Wasser wird dieselbe rasch zersetzt, unter Freiwerden von Chlorwasserstoff und Bildung von Toluylsäurekrystallen. Durch Alkohol wird sie zersetzt unter starker Wärmeentwickelung und Bildung von toluylsaurem Aethyl, welches sich isoliren läfst durch Zusatz von alkalihaltigem Wasser zu der alkoholischen Flüssigkeit, Trocknen mittelst Chlorcalcium und Rectificiren. Bei dem 
Zusammenreiben mit trockenem kohlensaurem Ammoniak erhitzt sich das Toluylchlorür stark und wird es zu Toluamid. Es siedet zwischen 214 und $216^{\circ}$; sein spec. Gewicht ist 1,175. Bei der Analyse ergab es :

\begin{tabular}{|c|c|c|c|c|}
\hline \multicolumn{3}{|c|}{ gefunden } & \multicolumn{2}{|c|}{ berechnet } \\
\hline Kohlenstoff & 61,83 & $\mathrm{C}_{16}$ & 96,0 & 62,13 \\
\hline Wasserstoff & 4,47 & $\mathrm{H}_{7}$ & 7,0 & 4,53 \\
\hline Chlor & 23,07 & $\mathrm{Cl}$ & 35,5 & 22,97 \\
\hline \multirow[t]{2}{*}{ Sauerstoff } & - & $\mathrm{O}_{2}$ & 16,0 & 10,37 \\
\hline & & & 154,5 & 100,00 \\
\hline
\end{tabular}

Cumosalicyl. - Das Cumylchlorür verhält sich gegen Salicylwasserstoff eben so wie die im Vorstehenden besprochenen Chlorverbindungen. In der Kälte findet keine Einwirkung statt; beim Erwärmen entwickelt sich reichlich Chlorwasserstoff und es bildet sich ein festes Product, welches in ähnlicher Weise, wie oben angegeben, gereinigt wird.

Das durch wiederholtes Umkrystallisiren gereinigte Cumosalicyl bildet farblose glänzende, leicht zerreibliche Prismen. Es ist unlöslich in kaltem Wasser, wenig löslich in siedendem Wasser, reichlicher löslich in Alkohol, namentlich in der Wärme. Aether löst es noch leichter. Es schmilat beim Erhitzen zu einer klaren, bei dem Erkalten krystallinisch erstarrenden Flüssigkeit. Festes Aetzkali und Kalilösung wirken auf es weder in der Kälte noch in der Hitze ein. Chlor, Brom und rauchende Salpetersäure wirken auf es ein, unter Bildung krystallisirbarer Producte.

Es ergab die Zusammensetzung :

\begin{tabular}{|c|c|c|c|c|c|}
\hline \multirow[b]{2}{*}{ Kohlenstoff } & \multicolumn{2}{|c|}{ gefunden } & \multirow[b]{2}{*}{$\mathrm{C}_{34}$} & \multicolumn{2}{|c|}{ berechnet } \\
\hline & 75,71 & 75,88 & & 204 & $\overline{76,1}$ \\
\hline Wasserstoff & 6,05 & 5,90 & $\mathrm{H}_{16}$ & 16 & 5,9 \\
\hline Sauerstoff & - & 一 & $\mathbf{O}_{6}$ & 48 & 18,0 \\
\hline & & & & 268 & 100,0 . \\
\hline
\end{tabular}


Anisosalicyl. - Wendet man Anisylchlorür an der Stelle des Cumylchlorürs an, so treten ganz ähnliche Erscheinungen wie die eben angegebenen auf. Das Einwirkungsproduct wird nach vorgängigem Waschen mit wässerigem Alkali und mit reinem Wasser in siedendern Alkohol gelöst; bei langsamem Erkalten der Lösung scheidet sich eine, meistens gelblich gefärbte, krystallinische Substanz aus. Durch Auspressen derselben zwischen Fliefspapier und Unkrystallisiren erhält man sie farblos.

Das auf diese Art dargestellte Anisosalicyl bildet farblose, durchsichtige, zerreibliche Prismen. Es ist in kaltem oder beifsem Wasser kaum löslich, aber ziemlich leicht löslich in Alkohol, namentlich in siedendem. Auch in Aether ist es löslich. Eben so wie die vorhergehenden Verbindungen wird auch das Anisosalicyl bei anhaltendem Kochen mit concentrirter Kalilösung nicht zersetzt. Es ergab die $\mathrm{Zu}-$ sammensetzung :

\begin{tabular}{|c|c|c|c|c|}
\hline \multicolumn{3}{|c|}{ gefunden } & \multicolumn{2}{|c|}{ berechnet } \\
\hline Kohlenstoff & 70,60 & $\mathrm{C}_{30}$ & $\overline{180}$ & 70,31 \\
\hline Wasserstoff & 4,77 & $\mathrm{H}_{12}$ & 12 & 4,68 \\
\hline Sauerstoff & 一 & $\underline{O_{8}}$ & 64 & 25,01 \\
\hline
\end{tabular}

Das Succinylchlorür verhält sich gegen das Salicylol eben so wie die vorhergehenden Chlorverbindungen; man erhält ein in farblosen Nadeln krystallisirendes Product, welches ich nicht analysirt habe, dessen Constitution indessen der der im Vorstehenden beschriebenen Verbindungen analog sein mufs. Sicher ist, dafs es wie diese durch Aetzkali nicht zersetzt wird.

Cyanosalicyl. - Da das Indigblan, indem es bei Einwirkung von Alkalihydraten das Wasser zersetzt, Salicylsäure bilden kann, und da andererseits die vom Indigo sich ableitenden wie die Salicyl-Verbindungen ein grofses Be- 
streben zeigen, in Phenylverbindungen überzugehen, so liefs sich hoffen, man könne das Salicylol zu Isatin umwandeln, da die Zusammensetzung beider Substanzen nur in der Art verschieden ist, als ob in dem Isatin sich 1 Aeq. Cyan an der Stelle von 1 Aeq. Wasserstoff in dem Salicylol befände. Es war also die Frage zu lösen, ob das Cyanosalicyl mit dem Isatin identisch oder nur isomer ist. Zur Entscheidung dieser Frage liefs ich Bromcyan, in wasserfreiem Alkohol gelöst, auf die Kaliumverbindung des Salicylols einwirken. Läfst man die Einwirkung bei gewöhnlicher Temperatur vor sich gehen, so sieht man bald Bromkalium sich abscheiden, während in dem Alkohol eine Substanz gelöst bleibt, die bei dem Verdunsten der Lösung in Form gelblicher Krystallschuppen erhalten wird. Letztere Verbindung verhält sich wie eine sehr schwache Base; sie kann sich mit Säuren vereinigen. Sie hat die Zusammensetzung des Isatins, aber Nichts von den Eigenschaften desselben. Die Analyse ergab : gefunden

\begin{tabular}{lccrrr} 
Kohlenstoff & 65,13 & & $\mathrm{C}_{16}$ & 96 & 65,30 \\
Wasserstoff & 3,51 & $\mathrm{H}_{5}$ & 5 & 3,40 \\
Stickstoff & - & $\mathrm{N}$ & 14 & 9,52 \\
Sauerstoff & - & & $\mathrm{O}_{4}$ & 32 & 21,78 \\
\cline { 4 - 6 } & & & & 147 & $100,00$.
\end{tabular}

Lälst man Bromcyan auf das mit der Kaliumverbindung des Salicylols isomere benzoësaure Kali einwirken, so ist das Resultat ein ganz anderes. Es findet eine reichliche Entwickelung von Kohlensäure statt, und ein flüchtiges 0 el von der Zusammensetzung und den Eigenschaften des Benzonitrils geht über, zugleich mit einer festen neutralen stickstoff halligen Substanz; der Rückstand bestebt aus Bromkalium. Die Einwirkung erfolgt ganz einfach entsprechend der Gleichung : $\mathrm{C}_{14} \mathrm{H}_{5} \mathrm{KO}_{4}+\mathrm{C}_{2} \mathrm{NBr}=2 \mathrm{CO}_{2}+\mathrm{BrK}+\mathrm{C}_{14} \mathrm{H}_{5} \mathrm{~N}$.

Diese Zersetzung ist nicht eine vereinzelt dastehende 
Thatsache; die der Benzoësäure homologen Säuren verhalten sich, wie ich gefunden habe, in ganz entsprechender Weise. So wird das cuminsaure Kali zu Cumonitril, entsprechend der Gleichung :

$$
\mathrm{C}_{20} \mathrm{H}_{11} \mathrm{KO}_{4}+\mathrm{C}_{2} \mathrm{NBr}=2 \mathrm{CO}_{2}+\mathrm{BrK}+\mathrm{C}_{20} \mathrm{H}_{11} \mathrm{~N} \text {. }
$$

Ich habe mich durch vergleichende Untersuchungen überzeugt, dafs die auf diese Art dargestellten Nitrile mit denjenigen, welche man durch Destillation der Ammoniaksalze der entsprechenden Säuren mit wasserfreier Phosphorsäure erhäll, vollkommen identisch sind.

\section{Derivate des Eugenols (der Nelkensäure).}

Da der bisher als Nelkensäure bezeichnete Bestandtheil des flüchtigen Nelkenöls sich, wie das Salicylol, direct mit Basen verbinden kann und er zu der Cuminsäure in einem ähnlichen Verhältnifs der Isomerie steht, wie es zwischen dem Salicylol und der Benzoësäure bestelit, so warf sich mir die Frage auf, ob nicht die Einwirkung organischer Chlorverbindungen auf jenen Bestandtheil des Nelkenöls analoge Resultate ergeben könne, wenn gleich das Salicylol und die s. g. Nelkensäure, für welche ich die Bezeichnung Eugenol vorschlage, Verschiedenheiten zeigen, die auf eine Verschiedenheit ihrer Constitution schliefsen lassen können. Das Salicylol bindet bei Mitwirkung von Alkalien 2 Aeq. Sauerstoff unter Bildung von Salicylsäure, während das Eugenol Nichts Derartiges zeigt; ferner wird das Salicylol bei Einwirkung von Chlor, Brom oder Salpetersäure zu bestimmten und krystallisirbaren Substitutionsproducten, während das Eugenol unter denselben Umständen zu klebrigen oder zähen, nicht flüchtigen Producten wird, welche sich nicht reinigen und somit auch keiner Analyse unterwerfen lassen.

Die Chlorüre des Benzoyls, des Toluyls, des Cumyls und des Anisyls verhalten sich gegen das Eugenol eben so wie gegen 
das Salicylol; in der Kälte tritt einfache Mischung ohne Wärmeentwickelung ein, bei höherer Temperatur findet Einwirkung statt unter reichlicher Entwickelung von Chlorwasserstoffsäure und Bildung eines zähen Products, das bei Behandlung mit Kali fest wird. Aus diesem Product Iassen sich durch Waschen desselben mit Wasser, Auspressen zwischen Fliefspapier und Auflösen in siedendem Alkohol krystallisirbare Substanzen erhalten, welche im Folgenden genauer beschrieben werden sollen.

Benzeugenyl. - Benzoylchlorür löst sich in Eugenol in der Kälte unverändert auf; selbst nach mehreren Stunden tritt keine Einwirkung ein. Erhitzt man aber die Mischung, so entwickelt sich bald Chlorwasserstoff in reichlicher Menge und die Substanz verdickt sich und färbt sich dunkelbraun. Sobald die Entwickelung von Chlorwasserstoff aufhört und die Masse nur noch hellbräunlichgelb gefärbt ist, verschlielst man das sie enthaltende Gefäls und läfst es erkalten. Bis zum andern Tage ist manchmal die zähe Flüssigkeit zu einer aus deutlichen Krystallen bestehenden Masse geworden, während in anderen Fällen der flüssige Zustand fortdauert; setzt man aber dem flüssigen Product concentrirte Kalilauge zu, um das im Ueberschusse vorhandene Eugenol oder Benzoylchlorür zu beseitigen, so tritt alsbald Festwerden der Masse ein. Die so erhaltene Substanz wird nun mit Wasser gewaschen, zwischen Flielspapier ausgeprefst und mit siedendem Alkohol behandelt, in welchem sie sich ziemlich leicht löst; aus dieser Lösung scheiden sich bei dem Erkalten oder Verdunsten deutliche Krystalle ab.

Die so erhaltene Substanz, welche ganz neutral ist und die ich als Benzeugenyl bezeichne, bildet nadelförmige Krystalle, die gewöhnlich hellamberfarbig sind, sich aber durch Destillation oder wiederholtes Umkrystallisiren ganz farblos erhalten lassen. Sie ist unlöslich in kaltem oder heifsem Annal. d. Chem, w. Pharm. CVIII. Bd. 3. Heft. 
Wasser, ziemlich löslich in siedendem Alkohol und auch in Aether. Sie schmilzt zwischen 50 und $55^{\circ}$ und siedet bei einer über $360^{\circ}$ liegenden Temperatur. Concentrirtes wässeriges Aetzkali wirkt auf das Benzeugenyl weder in der Kälte noch bei dem Kochen zersetzend ein. Anders aber verhält sich festes Aetzkali, welches auf das Benzeugenyl ähnlich wie auf zusammengesetzte Aetherarten oder neutrale Fette einwirkt und eine Verschiedenheit des Verhaltens des Benzeugenyls von dem der in analoger Weise aus Benzoylchlorïr und Salicylol entstehenden Substanz nachweist; das bei Einwirkung festen Aelzkali's entstehende Product löst sich nämlich leicht in Wasser, und Säuren scheiden daraus Eugenol und Benzoësäure ab. Brom und Salpetersäure wirken auf das Benzeugenyl energisch ein, wahrscheinlich unter Bildung von Substitutionsproducten, die ich indessen nicht näher untersucht habe.

Das Benzeugenyl ergab die Zusammensetzung :

\begin{tabular}{|c|c|c|c|c|c|c|}
\hline \multirow[b]{2}{*}{ Kohlenstoff } & \multicolumn{3}{|c|}{ gefunden } & & \multicolumn{2}{|c|}{ berechnet } \\
\hline & 75,88 & 75,69 & 75,59 & $\mathrm{C}_{34}$ & 204 & $7 \overline{6,12}$ \\
\hline Wasserstoff & 6,03 & 6,08 & 6,07 & $\mathrm{H}_{16}$ & 16 & 5,97 \\
\hline \multirow[t]{2}{*}{ Sauerstoff } & \multirow[t]{2}{*}{ 一 } & \multirow[t]{2}{*}{-} & \multirow[t]{2}{*}{-} & \multirow[t]{2}{*}{$\mathrm{O}_{6}$} & 48 & 17,91 \\
\hline & & & & & 268 & $100,00$. \\
\hline
\end{tabular}

Tolueugenyl. - Toluylchlorür zeigt gegen Eugenol ganz dasselbe Verhalten wie Benzoylchlorür. Das Einwirkungsproduct wird durch Behandlung mil Kali und Umkrystallisiren rein erhalten. Das so dargestellte Tolueugenyl bildet denen des Benzeugenyls sehr ähnliche nadelförmige Krystalle; es ist unlöslich in Wasser, ziemlich leicht löslich in siedendem Alkohol, noch löslicher in Aether. Festes Aetzkali verhält sich gegen es wie gegen Benzeugenyl; in der Hitze tritt vollständige Zersetzung ein, und Eugenol und Toluylsäure werden wieder gebildet.

Die Zusammensetzung des Tolueugenyls ist : 


\begin{tabular}{|c|c|c|c|c|c|}
\hline \multirow[b]{2}{*}{ Kohlenstoff } & \multicolumn{2}{|c|}{ gefunden } & \multirow[b]{2}{*}{$\mathrm{C}_{36}$} & \multicolumn{2}{|c|}{ berechnet } \\
\hline & 76,37 & 76,40 & & 216 & $7 \overline{76,59}$ \\
\hline Wasserstoff & 6,47 & 6,49 & $\mathrm{H}_{16}$ & 18 & 6,38 \\
\hline Sauerstoff & - & - & $\mathrm{O}_{6}$ & 48 & 17,03 \\
\hline & & & & 282 & 100,00 . \\
\hline
\end{tabular}

Cumeugenyl. - Das in ganz gleicher Weise aus Cumylchlorür und Eugenol sich bildende und eben so, wie die vorhergehenden Verbindungen, zu reinigende Cumeugenyl bildet glänzende farblose Tafeln, die schon bei geringer Erwärmung schmelzen und sich über $400^{\circ}$ verflüchtigen. Durch siedende Kalilösung wird es nicht angegriffen; durch festes Kalihydrat wird es in der Hitze unter Bildung von Eugenol und Cuminsäure zersetzt. Rauchende Salpetersäure wirkt auf das $\mathrm{Cu}-$ meugenyl lebhaft ein; es entsteht eine röthlichgelbe zähe Masse, in welcher Krystalle bemerkbar sind.

Das Cumeugenyl ergab die Zusammensetzung :

\begin{tabular}{|c|c|c|c|c|c|c|}
\hline \multirow[b]{2}{*}{ Kohlenstoff } & \multicolumn{3}{|c|}{ gefunden } & & \multicolumn{2}{|c|}{ berechnet } \\
\hline & 77,15 & 76,91 & $\overline{77,26}$ & $\mathrm{C}_{40}$ & 240 & $\overline{77,42}$ \\
\hline Wasserstoff & 7,24 & 7,12 & 7,06 & $\mathrm{H}_{22}$ & 22 & 7,09 \\
\hline \multirow[t]{2}{*}{ Sauerstoff } & \multirow[t]{2}{*}{-} & \multirow[t]{2}{*}{ - } & \multirow[t]{2}{*}{ - } & \multirow[t]{2}{*}{$\mathrm{O}_{6}$} & 48 & 15,49 \\
\hline & & & & & 310 & 100,00 \\
\hline
\end{tabular}

Es ist isomer mit der wasserfreien Cuminsäure, zeigt aber ganz andere Eigenschaften.

Anisylchlorür verhält sich gegen Eugenol eben so wie die im Vorhergehenden besprochenen Chlorverbindungen, und giebt ein krystallisirbares, in wässerigem Kali unlösliches Product, welches ich nicht analysirt habe, da aus seiner Bildungsweise genugsam hervorgeht, dafs seine Zusammensetzung der der vorstehend beschriebenen Verbindungen analog sein mufs.

Eugenäthyl. - Das Eugenol lälst sich nicht in derselben Weise wie die ihm isomere Cuminsäure - durch Auflösen in wasserfreiem Alkohol, Sätligen der Lösung mit Chlorwas- 
serstoffgas und Erhitzen derselben zum Sieden - ätherificiren, doch lälst sich das Eugenäthyl (nelkensaure Aethyl) in der Art darstellen, dafs man die Kaliumverbindung des Eugenols in verschlossenen Gefälsen mit Jodäthyl zersetzt; es entsteht dann Eugenäthyl unter gleichzeitiger Bildung von Jodkalium. Durch Waschen mit verdünntem wässerigem Alkali, Trocknen mittelst Chlorcalcium und Rectificiren gereinigt bildet dasselbe eine farblose neutrale, aromatisch und entfernt an Gewürznelken erinnernd riechende Flüssigkeit, welche gegen $240^{\circ}$ siedet, unlöslich in Wasser, leichtlöslich in Alkohol und in Aether ist, durch Chlor, Brom und rauchende Salpetersäure mit Heftigkeit unter Bildung nicht weiter untersuchter zäher Producte angegriffen wird. Die Zusammensetzung des Eugenäthyls ergab sich :

\begin{tabular}{lccrrr} 
& gefunden & & \multicolumn{2}{c}{ berechnet } \\
\cline { 4 - 6 } Kohlenstoff & $\mathbf{7 4 , 8 2}$ & $\mathrm{C}_{24}$ & 144 & $\mathbf{7 5 , 0 0}$ \\
Wasserstoff & 8,40 & $\mathrm{H}_{16}$ & 16 & 8,33 \\
Sauerstoff & - & $0_{4}$ & 32 & 16,67 \\
\cline { 3 - 5 } & & & & 192 & $100,00$.
\end{tabular}

Ueber die Aequivalentgewichte der einfachen Körper;

von J. Dumas*).

Obschon es mir noch nicht möglich war, die begonnene Revision der Aequivalentgewichte zu Ende zu führen, habe ich doch einige weitere Resultate erhalten, die mir beachtenswerth scheinen und, indem sie die früher ausgesproche-

*) Compt. rend. XLVI, 951. 